\title{
Predições da espectroscopia no infravermelho próximo podem determinar a digestibilidade e o consumo alimentar de cordeiros confinados
}

[Near-IR spectroscopy predictions can determine the digestibility and feed intake from feedlot lambs]

M.T.C. Almeida, T.R. Delphino, J.R. Paschoaloto, V. B. Carvalho, H. L. Perez, E.M.O. D'Aurea, A.P. D'Aurea, A.C. Homem Junior, V.R. Favaro, J.M.B. Ezequiel

Universidade Estadual Paulista - Unesp - Jaboticabal, SP

\section{RESUMO}

O presente trabalho teve como objetivo validar a eficácia do método de espectroscopia de reflectância do infravermelho proximal (NIRS) em predizer o consumo e a digestibilidade da MS e nutrientes de dietas para cordeiros confinados, em comparação ao método convencional de laboratório. Amostras das dietas fornecidas, sobras e fezes de 40 cordeiros Santa Inês foram submetidas a ambos os métodos e analisadas quanto aos teores de MS, MM, FDN, FDA, PB e EE, para futuros cálculos do consumo e da digestibilidade. A maioria dos coeficientes de correlação do modelo ficou na faixa de 0,66 a 0,81, mostrando-se adequados para a predição quantitativa. Os maiores coeficientes foram observados para teores de PB das dietas $(0,85)$ e sobras $(0,87)$. Foram encontradas altas correlações entre os métodos na predição do consumo (média de 0,93 ) e digestibilidade (média de 0,90 ) das dietas. As correlações entre as variáveis obtidas pela predição do NIRS e pelo método convencional foram adequadas, demonstrando que, independentemente do método, os teores nutricionais das dietas, das sobras e das fezes podem ser utilizados para calcular o consumo e a digestibilidade da MS e dos nutrientes das dietas para cordeiros confinados, sendo recomendado o método NIRS pela precisão, pela rapidez e pelo baixo custo em relação ao método convencional.

Palavras-chave: composição química, consumo, digestibilidade, NIRS, ovinos

\begin{abstract}
This research was carried out to validate the effectiveness of the Near-IR spectroscopy method in predicting intake and digestibility of DM and nutrients of diets for feedlot lambs, in order to compare with the conventional laboratory method. Samples of diets, ort,s and feces of forty Santa Ines lambs were subjected to both methods and analyzed for DM, MM, NDF, ADF, CP, and EE for future calculations for intake and digestibility. Most of the model's correlation coefficient was in the range of 0.66 to 0.81 , being suitable for quantitative prediction. Highest values for CP content of 0.85 (diets) and 0.87 (orts) were observed. There were high correlations between the methods to predicting intake (average values of 0.93) and digestibility (average values of 0.93). Correlations between the variables obtained by the prediction of NIRS and the conventional method were adequate, showing that regardless of the method, the nutritional content of diets, orts and feces can be used to calculate the intake and digestibility of DM and nutrients of lambs' diets. The Near-IR spectroscopy method is recommended for accuracy, speed and low cost compared to conventional method.
\end{abstract}

Keywords: chemical composition, digestibility, intake, NIRS, ovine

Recebido em 26 de outubro de 2016

Aceito em 20 de abril de 2017

E-mail: marcotulio695@yahoo.com.br 


\section{Almeida et al.}

\section{INTRODUÇÃO}

A avaliação da composição química dos alimentos é um dos principais pontos a se considerar para obter sucesso na nutrição animal. A busca pelo conhecimento do conteúdo nutricional de cada alimento, relacionado ao seu aproveitamento digestivo e metabólico, tem levado os pesquisadores a pesquisarem métodos analíticos cada vez mais eficazes, rápidos e de baixo impacto ambiental (Berchielli et al., 2011; Canesin et al., 2012).

Estudos sobre a composição dos alimentos datam do século XVII (Giuntini et al., 2006). Porém, segundo McMasters (1963), a primeira análise quantitativa em alimentos foi realizada em 1795, na Inglaterra, e, desde então, grandes avanços nas técnicas e novos métodos já foram reportados. Atualmente, entre os métodos comumente utilizados e tomados como padrões para a composição centesimal dos alimentos, está o método de Weende, de 1864, para teores de MS, MM, PB, FB e EE, e o método de Van Soest, de 1964, para teores de FDN e FDA, porém ambos com novas adaptações e unificações dos métodos (Silva e Queiroz, 2002; Detmann, et al., 2012; AOAC (Official..., 2016)). Contudo, apesar da padronização e aceitação mundial, as análises convencionais de laboratório demandam tempo, são susceptíveis a erros e possuem elevados custos operacionais, principalmente por utilizarem equipamentos e reagentes de alto custo, além de apresentarem alto poder de poluição ambiental, caso haja o descarte incorreto dos resíduos químicos das análises.

Nesse sentido, o método de análise por espectroscopia de reflectância do infravermelho proximal (NIRS) tem se mostrado uma alternativa ao método convencional, principalmente por permitir uma análise ampla dos constituintes dos alimentos sem a destruição das amostras e com alta precisão, rapidez, menor mão de obra e baixo poder de poluição ambiental, por não utilizar reagentes químicos, e a um baixo custo por unidade de análise (Deepa et al., 2016). O método NIRS é considerado uma tecnologia padrão e aceito como método oficial da AOAC, para a predição da FDA, PB (AOAC 989.03) e umidade (AOAC 991.01; Batten, 1998), além de apresentar resultados positivos na predição da ingestão e da digestibilidade (Reeves
III e Delwiche, 2003; Decruyenaere et al., 2009), degradabilidade ruminal (Antoniewicz et al., 1995; Hoffman et al., 1999) e componentes antinutricionais (Goodchild et al., 1998; Landau et al., 2006).

Diante de todo esse sucesso, muitos pesquisadores e empresas da área de nutrição animal têm optado por realizar as análises da composição nutricional dos alimentos pelo método NIRS. Porém, mesmo sendo uma técnica bem difundida, desenvolvida nas décadas de 1950 e 1960 e aplicada para análise de alimentos desde a década de 1970 (Norris et al., 1976), necessita de grande atenção e cuidado na utilização e calibração do aparelho, além de apresentar grande complexidade na escolha do tratamento dos dados gerados. Os procedimentos de calibração consomem tempo e necessitam de um grande número de amostras para a adequada formação do banco de dados. A utilização de um banco de dados pouco abrangente pode comprometer a confiabilidade das predições (Deepa et al., 2016), principalmente se esses dados forem utilizados para cálculos futuros de estimativas de consumo e digestibilidade de dietas para animais.

Assim, este trabalho teve como objetivo a validação da determinação do consumo e da digestibilidade da MS e de nutrientes por meio de dados preditos pelo NIRS, em comparação com os resultados obtidos pelo método convencional em laboratório, a partir de amostras de dietas, sobras e fezes de cordeiros confinados.

\section{MATERIAL E MÉTODOS}

O experimento foi conduzido nas instalações da Unidade Animal de Estudos Digestivos e Metabólicos e no Laboratório de Nutrição Animal (Lana), ambos pertencentes ao Departamento de Zootecnia da Universidade Estadual Paulista (FCAV/Unesp), Jaboticabal, SP, Brasil. Os procedimentos experimentais foram aprovados pela Comissão de Ética no Uso de Animais da Universidade Estadual Paulista (Protocolo Ceua 006558/12).

Para a coleta de amostras (dietas, sobras e fezes), foram utilizados 40 cordeiros machos, não castrados, da raça Santa Inês $(23,5 \mathrm{~kg} \pm 3,56 \mathrm{de}$ peso corporal e três meses de idade). Os animais permaneceram alojados individualmente em 
baias ripadas $\left(1,2 \mathrm{~m}^{2}\right)$, providas de cochos e bebedouros individuais. O período de adaptação dos animais às dietas, às instalações e ao manejo experimental foi de 14 dias.

As dietas experimentais foram formuladas segundo recomendações do NRC (Nutrient, 2007), para atender as exigências nutricionais de cordeiros pesando entre 20 e $30 \mathrm{~kg}$, para ganhos de peso entre 200 e $250 \mathrm{~g} / \mathrm{dia}$. O volumoso utilizado foi o feno de Tifton-85 (18\%) e o concentrado (82\%) composto por milho moído, farelo de girassol, casca de soja, glicerina bruta, glutenose, óleo de milho, ureia e suplemento mineral. Os animais foram alimentados duas vezes ao dia (sete horas e 16h), e o fornecimento foi ajustado diariamente para obter sobras em torno de $10 \%$ da ingestão anterior, a fim de garantir o consumo ad libitum de ração.

O consumo de MS foi avaliado durante todo o período experimental, por meio de pesagens diárias das quantidades das dietas fornecidas e das sobras. Para o consumo de nutrientes (MO, FDN, FDA, PB e EE), amostragens do fornecido e sobras foram coletadas no início (d14), meio (d28) e fim (d42) do período experimental. As coletas totais de fezes foram realizadas 24 horas após o fornecimento das dietas (d15, d29 e d43), durante três dias consecutivos, retirando-se uma amostra representativa de $20 \%$ do total diário para formar uma amostra composta no final de cada período de coleta. Todo material amostrado (dietas, sobras e fezes) foi identificado e congelado a $-20^{\circ} \mathrm{C}$ para posteriores processamentos e análises laboratoriais.

Com base nessas amostras, foram criadas amostras compostas de todo o período experimental para cada animal. Por fim, as amostras foram pré-secas em estufa de ventilação forçada a $55^{\circ} \mathrm{C}$ durante 72 horas e moídas em peneira de crivo de $1 \mathrm{~mm}$ para futuras análises químicas, pelo método convencional, e para análises físicas de predição, pelo método NIRS.

A concentração de matéria seca (MS) foi determinada por secagem do material em estufa a $105^{\circ} \mathrm{C}$ durante 24 horas (Official..., 1990; método 930.15), e o teor de cinzas por combustão completa em forno mufla a $600^{\circ} \mathrm{C}$ durante três horas (Official...,1990; método 942.05). As concentrações de fibra em detergente neutro (FDN) e da fibra em detergente ácido
(FDA) foram determinadas em analisador de fibra Ankom 220 ${ }^{\circledR}$ (Ankom Technology Corp., Fairport, NY) usando $\alpha$-amilase estável ao calor sem sulfito de sódio (Van Soest et al., 1991). A proteína bruta $(\mathrm{PB})$ foi determinada pelo método de Kjeldahl (Official..., 1990; método 981.10) e a concentração de extrato etéreo (EE) foi determinada pela extração com éter de petróleo, em aparelho de Soxhlet, durante seis horas (Official..., 1990; método 930.15).

Posteriormente, as amostras foram analisadas em duplicatas (dieta $(n=80)$, sobras $(n=80)$ e fezes $(\mathrm{n}=80))$ pelo método físico NIRS, utilizando-se um espectrômetro Perstorp Analytical, Silver Spring, MD, modelo 5000, acoplado a um computador equipado com software WinISI II versão 1.50 (Intrasoft International, University Park, PA).

Foram determinadas equações de predição e tratamento matemático específico dos espectros obtidos. Para a construção das curvas de calibração, o método de calibração multivariado utilizado foi o de regressão mínimos quadrados parciais (PLS), e utilizou-se o tratamento matemático direto. As leituras realizadas no aparelho NIRS foram por meio do módulo de reflectância, com intervalo de 1100 a $2500 \mathrm{~nm}$, tendo como resultado o espectro de $\log 1 / \mathrm{R}$ (reflectância) versus o comprimento de onda em nanômetros. Foi criado um banco de dados dos espectros obtidos das amostras contendo o valor conhecido da composição bromatológica, determinado pelo método convencional e pela curva espectral de cada uma das amostras analisadas no aparelho. Esses dados foram armazenados no software do aparelho para predizer os valores das futuras leituras das amostras.

Todas as amostras foram novamente analisadas no sistema NIRS para avaliar a habilidade de predição dos modelos construídos por meio de predição externa, a partir dos resultados anteriormente armazenados no banco de dados. Para a validação externa do modelo de previsão, foram obtidos gráficos com os espectros gerados para cada constituinte nutricional (MS, MM, FDN, FDA, PB e EE), sendo calculados os valores de SEP (erro de previsão do modelo) e $\mathrm{R}^{2}$ (coeficiente de correlação entre os pontos obtidos). Também foram realizadas análises de variância, utilizando-se o PROC GLM do pacote 


\section{Almeida et al.}

estatístico SAS 9.2 (SAS Institute, Inc, Cary, $\mathrm{NC}$ ), sendo as médias (método convencional versus método NIRS) comparadas pelo teste de Tukey a $5 \%$.

Por fim, para a validação da determinação matemática do consumo e da digestibilidade dos resultados preditos pelo NIRS em comparação aos resultados laboratoriais (método convencional), foram realizadas análises de regressão por meio do PROC REG do SAS 9.2, gerando uma equação linear e um coeficiente de correlação para cada variável estudada.

\section{RESULTADOS E DISCUSSÃO}

Na Fig. 1, estão apresentados os espectros NIR do conjunto das amostras (dietas $(1, n=80)$, sobras $(2, n=80)$ e fezes $(3, n=80))$ do logaritmo da refletância difusa $(\log 1 / \mathrm{R})$ no infravermelho próximo, região de 1100 a $2500 \mathrm{~nm}$. O método do PLS foi o que melhor se adequou para a seleção do modelo de regressão multivariada de calibração para os parâmetros avaliados.

Todos os espectros NIR demonstraram homogeneidade no perfil espectral, sem sinais diferenciados de absorção. Os espectros 1 e 3 (Fig. 1) obtiveram intervalo maior de desviopadrão das amostras analisadas. Porém, ao se avaliarem os resultados por intermédio da curva de validação, foi observado que não seria necessária a retirada de amostras dessa curva (outliers). Essa retirada de amostras se tornaria interessante caso algumas delas não representassem a população, fato que poderia prejudicar a predição das amostras.
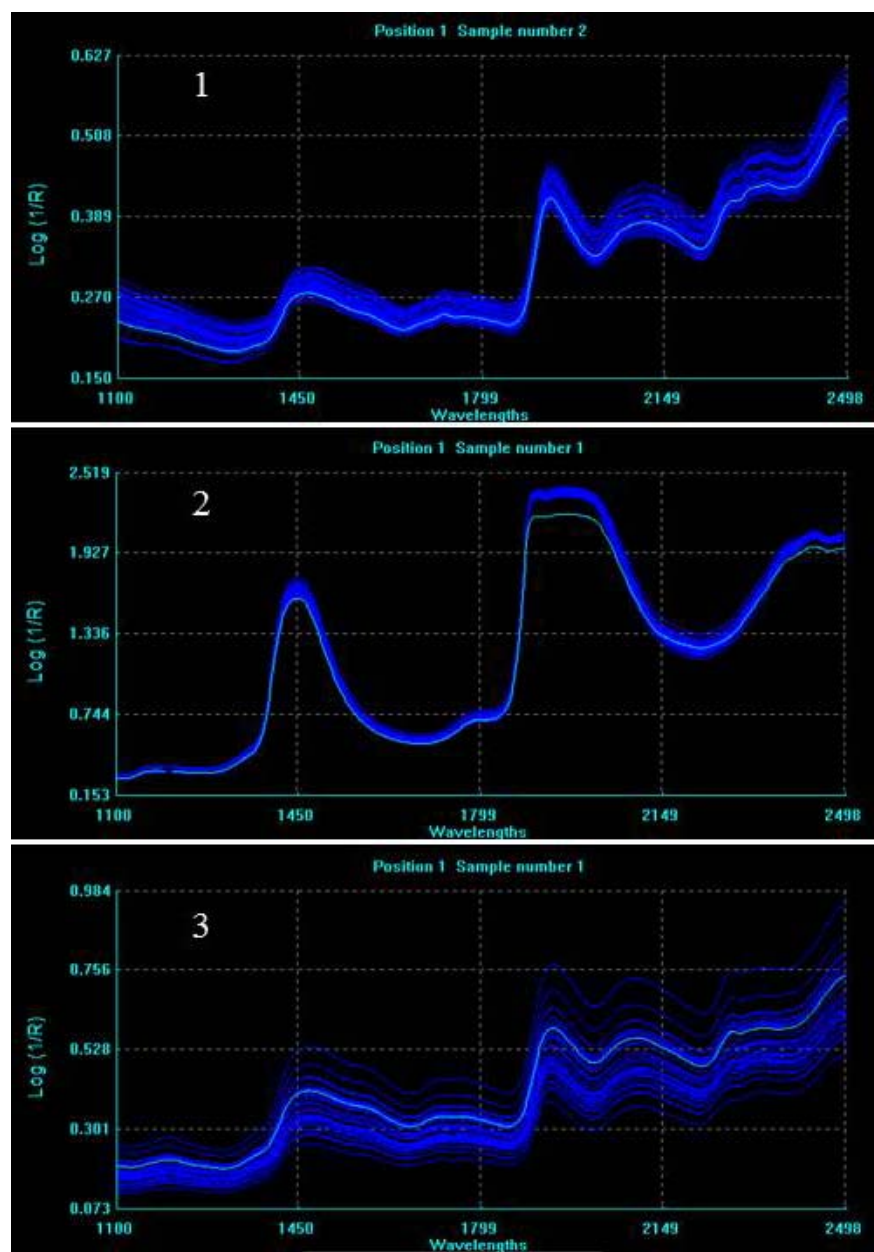

Figura 1. Espectros das amostras (dietas $(1, n=80)$, sobras $(2, n=80)$ e fezes $(3, n=80))$ do logaritmo da refletância difusa $(\log 1 / \mathrm{R})$ no infravermelho, região de 1100 a 2500nm. 
$\mathrm{Na}$ Tab. 1, notam-se os valores médios das análises laboratoriais e os resultados preditos para cada constituinte nutricional das dietas, sobras e fezes, assim como os resultados dos critérios considerados para o ajuste das curvas de calibração e equação da validação. A escolha do melhor modelo de regressão baseou-se na análise do menor erro de previsão do modelo para a validação externa (SEP), associado ao erro da calibração (EPC) e respectivo coeficiente de correlação da validação $\left(\mathrm{R}^{2}\right)$.

Tabela 1. Valores médios da composição nutricional das dietas, sobras e fezes, obtidos pelo método convencional e pelo método NIRS, e as variáveis da equação de validação

\begin{tabular}{|c|c|c|c|c|c|c|c|}
\hline \multirow{3}{*}{ Item } & \multicolumn{4}{|c|}{ Método } & \multirow{2}{*}{\multicolumn{3}{|c|}{ Convencional x NIRS }} \\
\hline & \multicolumn{2}{|c|}{ Convencional } & \multicolumn{2}{|c|}{ NIRS } & & & \\
\hline & Média & $\mathrm{EPM}^{\mathrm{A}}$ & Média & $\mathrm{EPC}^{\mathrm{B}}$ & $\mathrm{R}^{2}$ & $\mathrm{SEP}^{\mathrm{C}}$ & P-valor \\
\hline \multicolumn{8}{|c|}{ Composição das dietas } \\
\hline $\mathrm{MS}, \%$ & 78,05 & 0,33 & 78,31 & 0,30 & 0,80 & 0,22 & 0,566 \\
\hline MM, \% MS & 5,47 & 0,22 & 5,48 & 0,07 & 0,61 & 0,09 & 0,979 \\
\hline FDN, \% MS & 48,27 & 0,55 & 49,12 & 0,46 & 0,79 & 0,36 & 0,249 \\
\hline FDA, $\% \mathrm{MS}$ & 30,45 & 0,27 & 30,52 & 0,22 & 0,67 & 0,17 & 0,852 \\
\hline $\mathrm{PB}, \% \mathrm{MS}$ & 16,73 & 0,59 & 17,61 & 0,44 & 0,85 & 0,11 & 0,958 \\
\hline EE, \% MS & 2,39 & 0,09 & 2,39 & 0,01 & 0,74 & 0,14 & 0,245 \\
\hline \multicolumn{8}{|c|}{ Composição das sobras } \\
\hline MS, \% & 81,24 & 0,16 & 80,67 & 0,17 & 0,73 & 0,12 & 0,126 \\
\hline MM, \% MS & 6,39 & 0,05 & 6,25 & 0,08 & 0,58 & 0,35 & 0,099 \\
\hline FDN, $\% \mathrm{MS}$ & 47,02 & 0,46 & 49,00 & 0,39 & 0,61 & 0,31 & 0,074 \\
\hline FDA, $\% \mathrm{MS}$ & 31,84 & 0,34 & 31,59 & 0,36 & 0,74 & 0,25 & 0,230 \\
\hline $\mathrm{PB}, \% \mathrm{MS}$ & 12,69 & 0,20 & 12,73 & 0,22 & 0,87 & 0,02 & 0,936 \\
\hline EE, \% MS & 1,50 & 0,04 & 1,50 & 0,02 & 0,68 & 0,28 & 0,725 \\
\hline \multicolumn{8}{|c|}{ Composição das fezes } \\
\hline $\mathrm{MS}, \%$ & 31,01 & 0,05 & 30,78 & 0,04 & 0,71 & 0,13 & 0,317 \\
\hline MM, \% MS & 7,97 & 0,08 & 7,94 & 0,07 & 0,75 & 0,15 & 0,445 \\
\hline FDN, \% MS & 63,71 & 0,27 & 63,70 & 0,21 & 0,59 & 0,27 & 0,955 \\
\hline FDA, $\% \mathrm{MS}$ & 47,79 & 0,32 & 51,80 & 5,40 & 0,56 & 2,70 & 0,461 \\
\hline $\mathrm{PB}, \% \mathrm{MS}$ & 14,92 & 0,11 & 14,98 & 0,08 & 0,72 & 0,10 & 0,411 \\
\hline EE, \% MS & 1,33 & 0,03 & 1,32 & 0,02 & 0,66 & 0,02 & 0,410 \\
\hline
\end{tabular}

${ }^{\mathrm{A}}$ Erro-padrão da média.

${ }^{\mathrm{B}}$ Erro-padrão da calibração.

${ }^{\mathrm{C}}$ Erro-padrão da predição externa - Standard Error of Prediction.

Segundo Windham et al. (1989), resultados adequados são observados quando se têm baixos valores de SEP e altos valores de $\mathrm{R}^{2}$, e os resultados se tornam satisfatórios quando o valor do SEP for no máximo 1,3 vez maior que o valor de EPC. Assim, os modelos de predição dos constituintes nutricionais das dietas, sobras e fezes se mostraram adequados para a maioria dos constituintes, exceto para os teores de MM das sobras e fezes, que obtiveram, respectivamente, valores de SEP 4,4 e 2,1 vezes maiores que o EPC (Tab. 1).

Em relação aos coeficientes de correlação, valores próximos de um mostram maior capacidade de predição do modelo obtido (Burns e Ciurczak, 2007). Segundo Almeida (2009), valores entre 0,66 e 0,81 são aceitáveis para predições quantitativas, entre 0,83 e 0,90 aceitáveis para muitas aplicações e entre $0,92 \mathrm{e}$ 0,96 indicados para determinar controle de qualidade. Neste trabalho, observa-se que $72,22 \%$ das predições do modelo ficaram na faixa de 0,66 a 0,81 (Tab. 1), mostrando-se satisfatório para a maioria dos constituintes nutricionais, exceto para os teores de MM das dietas $\left(\mathrm{R}^{2}=0,61\right), \mathrm{MM}$ das sobras $\left(\mathrm{R}^{2}=0,58\right)$ e FDN e FDA das fezes $\left(\mathrm{R}^{2}=0,59\right.$ e $\mathrm{R}^{2}=0,56$, respectivamente). Os maiores valores de coeficiente de correlação foram observados para os teores de PB das dietas e sobras $(0,85$ e 0,87 , respectivamente). 
Alguns autores têm verificado que os modelos preditos pela técnica do NIRS para os teores de MM, FDN e FDA são insatisfatórios (Fontaneli et al., 2002; Shenk e Westerhaus, 1995; Pires e Pates, 1998). Em relação aos minerais, sabe-se que o método NIRS não se mostra apropriado para predição desse constituinte, por subestimar os valores. Isso acontece porque formas iônicas e salinas não absorvem energia na região do infravermelho próximo, uma vez que absorve energia por moléculas orgânicas, principalmente de $\mathrm{H}$ vinculado com $\mathrm{C}, \mathrm{O}$ e $\mathrm{N}$ (Shenk e Westerhaus, 1994). Porém, uma análise de mineral poderia ser satisfatória se estivesse associada a uma substância orgânica (Marten et al., 1989), fato que se justifica para este trabalho, entre as semelhanças de valores encontrados nos dois métodos analisados $(\mathrm{P}>0,05$, convencional versus NIRS).

Em relação às predições de FDA e FDN, existem poucas substâncias que poderiam interferir nesses comprimentos de onda e superestimar ou subestimar os valores dessas frações, pois se sabe que o método NIRS utiliza o comprimento de onda da ligação $\mathrm{C}=\mathrm{O}$ e $\mathrm{H}-\mathrm{C}=\mathrm{O}$ para relacionar com os carboidratos. Predições dos teores de FDN e FDA se tornam insatisfatórias somente se houver erros na calibração e no preparo, ou quando houver um número insuficiente de amostras. O sistema de análise NIRS é considerado uma tecnologia padrão e aceito como um método oficial da AOAC para predição da FDA, PB (AOAC 989.03) e umidade (AOAC 991.01; Batten, 1998). Nesse sentido, para este trabalho, o erro no modelo para predição de FDN e FDA poderia estar nas análises convencionais. As análises convencionais de FDN e FDA foram realizadas em aparelho ANKON para minimizar os erros obtidos pelo método de Van Soest (Berchielli et al., 2001). Porém, mesmo assim, possivelmente ocorreram erros nas análises, seja no preparo de amostras, filtração, lavagem, seja na pesagem das amostras. Contudo, apesar de alguns dados insatisfatórios do modelo de predição, não foram observadas diferenças $(\mathrm{P}>0,05)$ entre as médias dos dois métodos (convencional versus NIRS, Tab. 1) para nenhum dos constituintes nutricionais estudados, comprovando a eficácia do método NIRS.

Após a verificação da falta de diferença estatística entre os dois métodos, foram analisadas as correlações das variáveis de consumo e digestibilidade obtidas matematicamente pelos dois métodos. Em relação às variáveis de consumo, foram observados altos coeficientes de correlação entre os métodos (MS, $\mathrm{R}^{2}=0,99 ; \mathrm{MO}, \mathrm{R}^{2}=0,98 ; \mathrm{FDN}$, $\mathrm{R}^{2}=0,99 ; \quad F D A, \quad \mathrm{R}^{2}=0,99 ; \quad \mathrm{PB}, \mathrm{R}^{2}=0,91 ; \quad \mathrm{EE}$, $\mathrm{R}^{2}=0,73$, Fig. 2). Apesar de o consumo de EE ter apresentado o menor coeficiente, ainda está dentro do considerado adequado para predições quantitativas (Almeida, 2009).

Para as digestibilidades de MS, MO, FDN, PB e EE (\%), também foram observadas altas correlações entre os métodos $(0,99 ; 0,99 ; 0,97$; 0,84 e 0,70 , respectivamente), exceto para digestibilidade da FDA, com correlação de 0,46 (Fig. 3). Esse fato pode ser devido ao erro de metodologia supracitado, desencadeando erros nos cálculos da digestibilidade.

Os teores de MM obtidos nos dois métodos foram utilizados para correções dos teores de MO das amostras (dietas, sobras e fezes), para, assim, serem utilizados nos cálculos de consumo e digestibilidade da MO. Como o teor de MM é relativamente menor em relação à $\mathrm{MS}$, as diferenças observadas para este nutriente nas equações de validação não atrapalharam a mensuração das variáveis de consumo e digestibilidade (Fig. 2 e 3).

Por fim, as correlações entre as variáveis obtidas pela predição do NIRS e pelo método convencional foram satisfatórias, demonstrando que, independentemente do método utilizado, os teores dos nutrientes das dietas, sobras e fezes podem ser utilizados para calcular o consumo e a digestibilidade da MS e dos nutrientes (MO, FDN, PB e EE) das dietas de cordeiros confinados. 

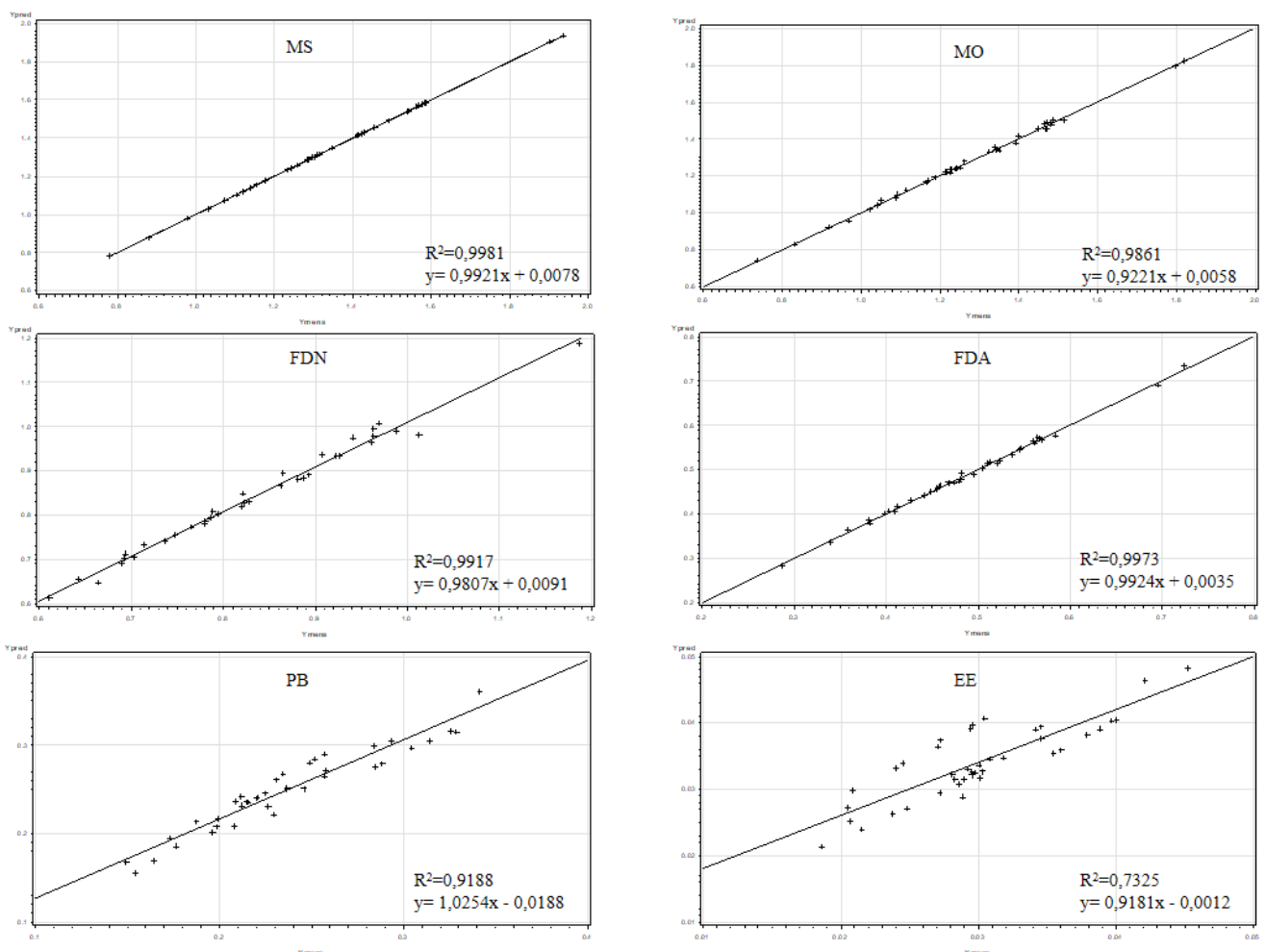

Figura 2. Consumo de MS e nutrientes (kg/dia), determinados matematicamente por valores obtidos pelo método convencional e por valores preditos pelo método NIRS.
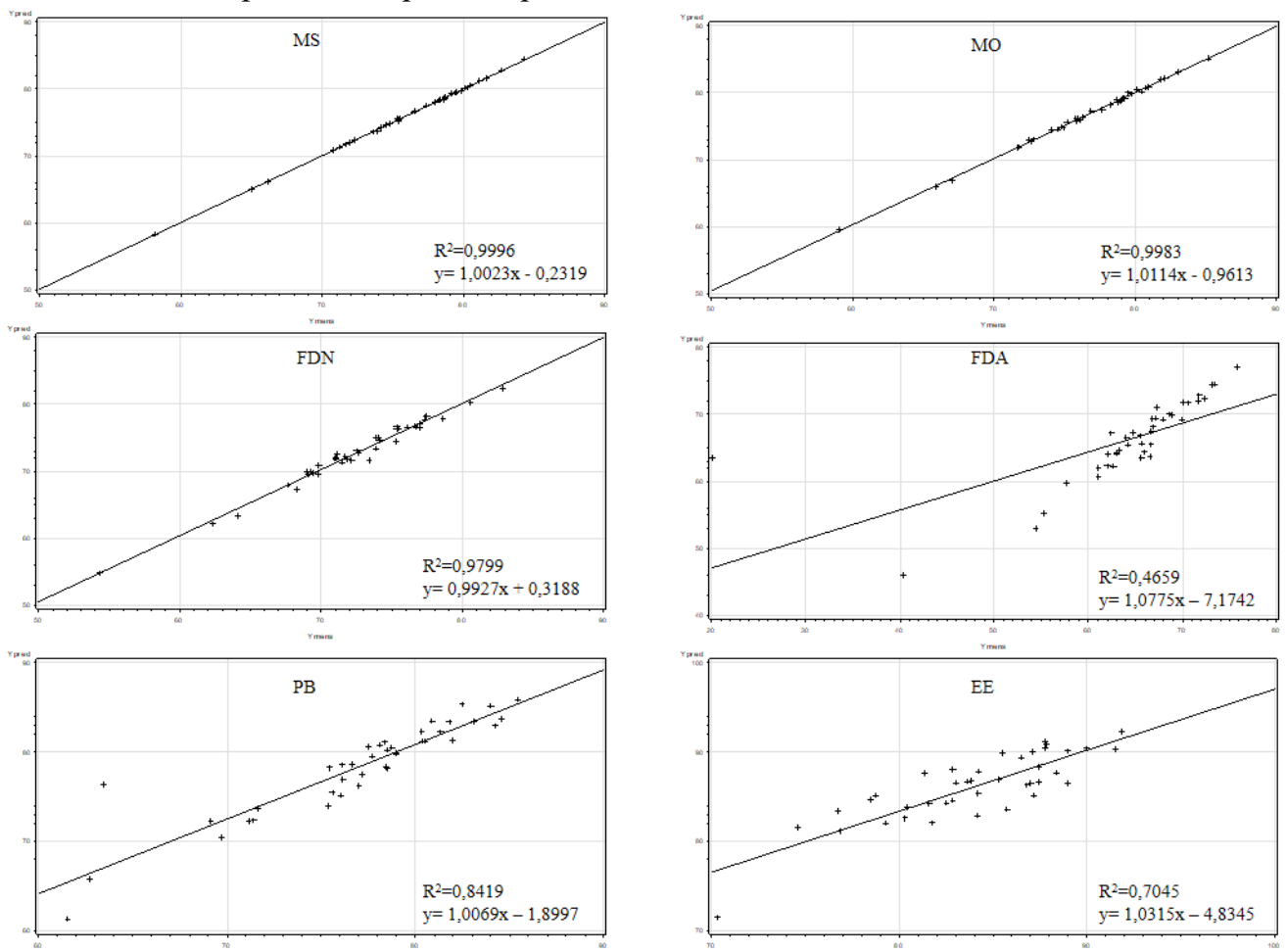

Figura 3. Digestibilidade da MS e nutrientes (\%), determinados matematicamente por valores obtidos pelo método convencional e por valores preditos pelo método NIRS. 


\section{CONCLUSÕES}

O método NIRS apresentou predições satisfatórias para cálculo das estimativas de consumo e digestibilidade da MS e dos nutrientes, sendo recomendado para o uso em experimentação animal por ser um método preciso, rápido e de baixo custo.

\section{AGRADECIMENTOS}

Às empresas Caramuru SA, pela doação da glicerina bruta, e Cargill, pelo fornecimento da glutenose.

\section{REFERÊNCIAS}

ALMEIDA, F. Espectroscopia de infravermelho próximo com transformada de fourier (ft-nir) na caracterização de farinhas para alimentação pueril. 2009. 73f. Dissertação (Mestrado em Engenharia Biológica) - Instituto Superior Técnico, Universidade Técnica de Lisboa, Lisboa, POR.

ANTONIEWICZ, A.M.; KOWALCZYK, J.; KANSKI, J. et al. Rumen degradability of crude protein of dried grass and lucerne forage measured by in sacco incubation and predicted by near infrared spectroscopy. Anim. Feed Sci. Technol., v.54, p.203-216, 1995.

BATTEN, G.D. Plant analysis using near infrared reflectance spectroscopy: the potential and the limitations. Anim. Prod. Sci., v.38, p.697-706, 1998.

BERCHIELLI, T.T.; GARCIA, A.D.V; OLIVEIRA, S.D. Principais técnicas de avaliação aplicadas em estudo de nutrição. In: BERCHIELLI, T.T.; PIRES, A.V.; OLIVEIRA, S.G. (Eds.). Nutrição de ruminantes. 2.ed. Jaboticabal: Funep, 2011. p.565-600.

BERCHIELLI, T.T.; SADER, A.D.O.; TONANI, F.L. et al. Avaliação da determinação da fibra em detergente neutro e da fibra em detergente ácido pelo sistema ANKOM. Rev. Bras. Zootec., v.30, p.572-578, 2001.

BURNS, D.A.; CIURCZAK, E.W. (Eds.). Handbook of near-infrared analysis. Boca Raton: CRC Press, 2007. 808p.
CANESIN, R.C.; FIORENTINI, G.; BERCHIELLI, T.T. Inovações e desafios na avaliação de alimentos na nutrição de ruminantes. Rev. Bras. Saúde Prod. Anim., v.13, p.938-953, 2012.

DECRUYENAERE, V.; LECOMTE, P.; DEMARQUILLY, C. et al. Evaluation of green forage intake and digestibility in ruminants using near infrared reflectance spectroscopy (NIRS): developing a global calibration. Anim. Feed Sci. Technol., v.148, p.138-156, 2009.

DEEPA, K.; SENTHILKUMAR, S.; KALPANA, K. et al. NIRS in animal sciences. Inter. J. Sci., v.5, p.605-610, 2016.

DETMANN, E.; SOUZA, M.D.; VALADARES FILHO, S.D.C. et al. (Eds.). Métodos para análise de alimentos. [Lavras]: INCT, 2012. $214 \mathrm{p}$.

FONTANELI, R.S.; DURR, J.W.; SCHEFFERBASSO. S.M. et al. Validação do método da reflectância no infravermelho proximal para análise de silagem de milho. Rev. Bras. Zootec., v.31, p.594-598, 2002.

GIUNTINI, E.B.; LAJOLO, F.M.; MENEZES, E.W. Composição de alimentos: um pouco de história. Arch. Latinoam. Nutr., v.56, p.295-303, 2006.

GOODCHILD, A.V.; EL HARAMEIN, F.J.; EL MONEIM, A.A. et al. Prediction of phenolics and tannins in forage legumes by near infrared reflectance. J. Near Infrared. Spectrosc., v.6, p.175-182, 1998.

HOFFMAN, P.C.; BREHM, N.M.; BAUMAN, L.M. et al. Prediction of laboratory and in situ protein fractions in legume and grass silages using near-infrared reflectance spectroscopy. $J$. Dairy Sci., v.82, p.764-770, 1999.

LANDAU, S.; GLASSER, T.; DVASH, L. Monitoring nutrition in small ruminants with the aid of near infrared reflectance spectroscopy (NIRS) technology: a review. Small Ruminant. Res., v.61, p.1-11, 2006.

MARTEN, G.C.; SHENK, J.S.; BARTON, F.E. (Eds.). Near infrared reflectance spectroscopy (NIRS): analysis of forage quality. Springfield: Agriculture handbook, 1989. 110p.

MCMASTERS, V. History of food composition tables of the word. J. Am. Diet. Assoc., v.43, p.442-450, 1963. 
NORRIS, K.H.; BARNES, R.F.; MOORE, J.E.; SHENK, J.S. Predicting forage quality by infrared replectance spectroscopy. J. Anim. Sci., v.43, p.889-897, 1976.

NUTRIENT requirements of small ruminant. Washington: National Academy of Sciences, 2007. 362p.

OFFICIAL methods of analysis. 15.ed. Gaithersburg: AOAC, 1990. v.1, 684p.

OFFICIAL methods of analysis. 20.ed. Gaithersburg: AOAC, 2016. v.1, 3172p.

PIRES, F.F.; PRATES, E.R. Uso da técnica da espectrofotometria de refletância no infravermelho proximal (NIRS) na predição da composição química da alfafa. Rev. Bras. Zootec., v.27, p.1076-1081, 1998.

REEVES III, J.B.; DELWICHE, S.R. SAS ${ }^{\circledR}$ partial least squares regression for analysis of spectroscopic data. J. Near Infrared Spectrosc., v.11, p.415-431, 2003.

SHENK, J.S.; WESTERHAUS, M.O. (Eds.). Routine operation, calibration and network system management software for near infrared instruments. Intrasoft International: Port Matilda, NIRS3++, v. 3.10, p. 100, 1995.
SHENK, J.S.; WESTERHAUS, M.O. The application of near infrared reflectance spectroscopy (NIRS) to forage analysis. In: MERTENS, D.R.; FAHEY, G.C. Forage quality, evaluation, and utilization. Madison: American Society of Agronomy, 1994. p.406-449.

SILVA, D.J.; QUEIROZ, A.C. (Eds.). Análise de alimentos: métodos químicos e biológicos. Viçosa: Universidade Federal de Viçosa, 2002. $166 \mathrm{p}$.

VAN SOEST, P.V.; ROBERTSON, J.B.; LEWIS, B.A. Methods for dietary fiber, andnonstarchpolyssacarides in relations to animal nutrition. J. Dairy Sci., v.74, p.35833597. 1991.

WINDHAM, W.R.; MERTENS, D.R.; BARTON II, F.E. 1. Protocol for NIRS calibration: sample selection and equation developement and validation. In: MARTEN, G.C.; SHENK, J.S.; BARTEN II, Springfield: F.E. (Eds.). Near infrared reflectance spectroscopy (NIRS): analysis of forage quality. USDA, 1989. p.96-103. 